\title{
Circulating and disseminated tumor cells from breast cancer patient-derived xenograft-bearing mice as a novel model to study metastasis
}

Mario Giuliano 1,2, Sabrina Herrera ${ }^{1}$, Pavel Christiny ${ }^{3}$, Chad Shaw $^{4,5}$, Chad J Creighton ${ }^{4,6}$, Tamika Mitchell ${ }^{1}$, Raksha Bhat ${ }^{3}$, Xiaomei Zhang ${ }^{1}$, Sufeng Mao ${ }^{1}$, Lacey E Dobrolecki ${ }^{1}$, Ahmed Al-rawi ${ }^{3}$, Fengju Chen ${ }^{4}$, Bianca M Veneziani ${ }^{7}$, Xiang H-F Zhang ${ }^{1,4,6,8}$, Susan G Hilsenbeck ${ }^{1,4,6}$, Alejandro Contreras ${ }^{1,4,9}$, Carolina Gutierrez ${ }^{1,4,9}$, Rinath M Jeselsohn ${ }^{1,10}$, Mothaffar F Rimawi ${ }^{1,4,6}$, C Kent Osborne ${ }^{1,4,6}$, Michael T Lewis ${ }^{1,4,11}$, Rachel Schiff ${ }^{1,4,6,8^{*}}$ and Meghana V Trivedi $1,3,4,6,12^{*}$

\begin{abstract}
Introduction: Real-time monitoring of biologic changes in tumors may be possible by investigating the transitional cells such as circulating tumor cells (CTCS) and disseminated tumor cells in bone marrow (BM-DTCs). However, the small numbers of CTCs and the limited access to bone marrow aspirates in cancer patients pose major hurdles. The goal of this study was to determine whether breast cancer (BC) patient-derived xenograft (PDX) mice could provide a constant and renewable source of CTCS and BM-DTCS, thereby representing a unique system for the study of metastatic processes.

Methods: CTCs and BM-DTCs, isolated from BC PDX-bearing mice, were identified by immunostaining for human pan-cytokeratin and nuclear counterstaining of red blood cell-lysed blood and bone marrow fractions, respectively. The rate of lung metastases (LM) was previously reported in these lines. Associations between the presence of CTCS, BM-DTCs, and LM were assessed by the Fisher's Exact and Cochran-Mantel-Haenszel tests. Two separate genetic signatures associated with the presence of CTC clusters and with lung metastatic potential were computed by using the expression arrays of primary tumors from different PDX lines and subsequently overlapped to identify common genes.
\end{abstract}

Results: In total, 18 BC PDX lines were evaluated. CTCs and BM-DTCs, present as either single cells or clusters, were detected in $83 \%$ (15 of 18) and $62.5 \%$ (10 to16) of the lines, respectively. A positive association was noted between the presence of CTCS and BM-DTCS within the same mice. LM was previously found in 9 of 18 (50\%) lines, of which all nine had detectable CTCs. The presence of LM was strongly associated with the detection of CTC clusters but not with individual cells or detection of BM-DTCs. Overlapping of the two genetic signatures of the primary PDX tumors associated with the presence of CTC clusters and with lung metastatic potential identified four genes (HLA-DPIA, GJA1, PEG3, and XIST). This four-gene profile predicted distant metastases-free survival in publicly available datasets of early BC patients.

Conclusion: This study suggests that CTCs and BM-DTCs detected in BC PDX-bearing mice may represent a valuable and unique preclinical model for investigating the role of these rare cells in tumor metastases.

\footnotetext{
* Correspondence: rschiff@bcm.edu; mtrivedi@UH.EDU

'Lester and Sue Smith Breast Center, Baylor College of Medicine, Houston,

TX, USA

${ }^{3}$ Department of Pharmacy Practice and Translational Research, University of

Houston, Houston, TX, USA

Full list of author information is available at the end of the article
}

\section{Biomed Central}

(c) 2015 Giuliano et al.; licensee BioMed Central. This is an Open Access article distributed under the terms of the Creative Commons Attribution License (http://creativecommons.org/licenses/by/4.0), which permits unrestricted use, distribution, and reproduction in any medium, provided the original work is properly credited. The Creative Commons Public Domain Dedication waiver (http://creativecommons.org/publicdomain/zero/1.0/) applies to the data made available in this article, unless otherwise stated. 


\section{Introduction}

Circulating tumor cells (CTCs) are cancer cells originating from either a primary or metastatic tumor and circulating freely in the peripheral blood [1]. It has been proposed that the spread of a primary tumor through the bloodstream as CTCs is a critical step in tumor metastasis $[2,3]$. In breast cancer (BC), CTCs can be detected in patients at early stages or late stages of disease with overt metastases [4-6]. Many studies have shown that the detection of CTCs may help to predict the outcome in patients with different types of cancers. In particular, the enumeration of CTCs before starting systemic treatment is associated with clinical outcome in both metastatic and non-metastatic $\mathrm{BC}$ patients $[4,6,7]$. Furthermore, CTC count evaluated at different time points during systemic treatment is a reliable surrogate marker of treatment response [8-12]. Preliminary studies have suggested that selecting therapies based on molecular characteristics of CTCs may improve treatment outcomes in patients [13-15]. Because CTCs are found in circulation as a collectable fraction that is representative of the tumor, they may provide an ideal model to study the biology of the tumor at various intervals before and during treatment [16].

Interestingly, the presence of CTCs has been found to correlate with the presence of disseminated tumor cells in the bone marrow (BM-DTCs) in BC patients $[17,18]$. Similar to CTCs, BM-DTCs play a crucial role in the metastatic cascade as the earliest detectable form of micrometastatic disease and potential precursors of overt metastases [19]. Notably, several studies have shown that persistence of BM-DTCs after therapy predicts a higher risk of relapse in BC patients $[20,21]$. Therefore, BM-DTCs represent an additional tool for studying the metastatic process in its initial stage.

Despite the evidence to support the roles of CTCs and BM-DTCs in studying tumor biology and predicting treatment response, routine clinical and preclinical use of these cells is challenging because of multiple factors. First, CTCs are present in small numbers in only $10 \%$ to $50 \%$ of BC patients [22-24]. Therefore, they cannot be isolated in sufficient numbers from a small volume of blood from most patients. Similarly, a longitudinal study of BM-DTCs is impractical because of limited access to bone marrow aspirates and the small number of DTCs that can be enriched from aspirates of standard volume. In addition, commonly used methods to detect CTCs use antibodies against epithelial cell markers and exclude identification of tumor cells with mesenchymal properties. This is especially problematic, as the cells that have undergone epithelial-to-mesenchymal transition (EMT) may play an essential role in the metastatic process [25].

To address these major challenges, we aimed to determine whether $\mathrm{BC}$ patient-derived xenograft (PDX)-bearing mice could provide a constant and renewable source of CTCs and BM-DTCs as a unique system to study the molecular changes responsible for tumor progression and metastases. Here, we report the detection of human CTCs and BM-DTCs in various BC PDX mice models [26]. To identify the PDX lines with high numbers of CTCs and BM-DTCs, we screened a total of 18 lines representing different molecular subclasses of BC. Further, we evaluated the association of CTC detection with the presence of BMDTCs and with the lung metastatic potential of these PDX lines. Finally, we determined the predictive value of a genetic profile computed from the primary tumors of various PDX lines that was associated with the presence of CTC clusters and lung metastatic potential.

\section{Methods}

\section{BC PDX mouse models}

BC PDX mouse models were established in Dr. Michael Lewis' laboratory at the Lester and Sue Smith Breast Center in Baylor College of Medicine. Methods used to establish these PDX models have been recently reported [26]. Mice transplanted with tumors from passages 2 through 11 were used for our studies. Animal care for the mice bearing the BC PDX tumors, as well as ageand gender-matched control mice, was in accordance with the NIH Guide for the Care and Use of Experimental Animals with approval from the Baylor College of Medicine Institutional Animal Care and Use Committee.

In brief, mammary fat pad epithelium was surgically cleared from 3- to 4-week-old SCID/Beige female mice. Subsequently, fresh breast tumor fragments collected directly from patients were orthotopically transplanted into the cleared mammary fat pads. When tumor size reached $1,000 \mathrm{~mm}^{3}$, mice were killed, and tumors were re-transplanted into additional mice up to 11 passages. Importantly, the primary and serially passaged PDXs have shown genomic, proteomic, phenotypic, and histologic consistency with the tumor of origin, and they are also genetically and proteomically stable across multiple transplant generations [26].

\section{Detection of CTCs, BM-DTCs, and lung metastases}

After anesthesia, peripheral blood (500 to $700 \mu \mathrm{l})$ was collected from the vena cava inferior of each animal, which was then killed by cervical dislocation. Tibias, femurs, and hip-bones were collected, and bone marrow was flushed from the bones by using phosphate-buffered saline (PBS) supplemented with $2 \mathrm{~m} M$ ethylenediaminetetraacetic acid (EDTA). Whole blood was processed within 1 hour of collection to lyse red blood cells (RBCs) by incubation with ammonium chloride (StemCell Technologies, Vancouver, $B C$, Canada) per manufacturer's protocol.

RBC-depleted cells and bone marrow cells were then washed twice with PBS at room temperature, pelleted, 
and fixed with $10 \%$ neutral buffered formalin for 3 hours at room temperature. Cell pellets were embedded in paraffin and cut in consecutive sections of $5-\mu \mathrm{m}$ thickness. Five consecutive sections of every 15 sections were stained by using immunohistochemistry (IHC) with antihuman pan-cytokeratin (clone AE1/AE3 against cytokeratins 1-8, 10, 13-16, and 19; Source: Dako, Carpinteria, CA, USA) and nuclear counterstain (hematoxylin). CTCs and BM-DTCs were identified as cytoplasmic human pancytokeratin-positive and nuclear counterstain-positive cells. A CTC or BM-DTC cluster was defined as a group of two or more CTCs or BM-DTCs, respectively. At least two PDX-bearing mice were tested per line (range, 2 to 7), and in total, five age-matched non-tumor-bearing female mice were used as controls. Lung metastases (LMs) were identified by IHC in these PDX lines and were reported in the previous study [26].

\section{Genetic signature of primary tumors associated with CTC clusters and LM}

The following nine of 18 screened lines had published Affymetrix gene expression data (GEO:GSE46106), and at least three mice screened for CTCs (BCM-3107, BCM-3204, BCM-3561, BCM-3613, BCM-3887, BCM3963, BCM-4272, BCM-4664, BCM-4888) [26]. Of these nine lines, CTC clusters were present in three lines and LM were detected in six lines (Table 1). To identify differential genetic signatures corresponding to CTC clusters and to LM, analysis of variance followed by $\mathrm{t}$ tests were performed by applying linear modeling to our microarray experiments by using the Linear Models for Microarray Data (LIMMA) method, and subsequent manual curation was used, as described previously [27]. A genetic profile overlapping these two genetic signatures was derived and interrogated for prediction of distant metastasis-free survival in publicly available datasets [28].

\section{Statistics}

The number of CTCs was reported per 20,000 nucleated cells $(\approx 20 \mu \mathrm{l}$ of blood). This was based on our initial testing of $>20$ representative mice from nine PDX models showing that the average number of RBC-depleted nucleated cells was 20,000 per $20 \mu \mathrm{l}$ of blood collected. The DTC count was reported as number of DTCs per 2 million bone marrow cells. In addition, the CTC and BMDTC detection rates were calculated per each PDX line as the ratio of the number of mice with one or more CTCs and DTCs, respectively, and the number of mice tested. Lung metastatic rate was previously reported as the ratio of the mice affected by metastases and the number of mice tested per each line [26]. The association between the presence of CTCs and BM-DTCs was evaluated within the same mice by using Fisher's Exact test and CochranMantel-Haenszel test to adjust for different PDX lines.

Table 1 Identification of CTCs and BM-DTCs in BC PDX lines and previously reported presence of LM

\begin{tabular}{|c|c|c|c|c|c|}
\hline PDX line & $\begin{array}{l}\text { CTC detection } \\
\text { rate }(\%)\end{array}$ & $\begin{array}{l}\text { Number of CTCs per } 20 \mathrm{~K} \text { nucleated } \\
\text { cells }(\approx 20 \mu \mathrm{l} \text { of blood })\end{array}$ & $\begin{array}{l}\text { BM-DTC detection } \\
\text { rate }(\%)\end{array}$ & $\begin{array}{l}\text { Number of BM-DTCs per } \\
2 \times 10^{6} \text { BM cells }\end{array}$ & LM rate [26] (\%) \\
\hline BCM-3107* & $1 / 4(25)$ & 7 & $1 / 2(50)$ & 1 & 0 \\
\hline BCM-3143 & $0 / 2(0)$ & 0 & $0 / 2(0)$ & 0 & 0 \\
\hline BCM-3204* & $2 / 4(50)^{C l}$ & $3-43$ & $0 / 2(0)$ & 0 & 29 \\
\hline BCM-3561* & $2 / 4(50)$ & 1 & ND & ND & 0 \\
\hline BCM-3613* & $1 / 3(33)$ & 7 & $0 / 2(0)$ & 0 & 24 \\
\hline BCM-3807 & $0 / 4(0)$ & 0 & $1 / 2(50)$ & 29 & 0 \\
\hline BCM-3887* & $3 / 4(75)^{C l}$ & $3-92$ & $1 / 5(20)$ & $<1$ & 14 \\
\hline BCM-3963* & $1 / 6(17)$ & 7 & $0 / 4(0)$ & 0 & 14 \\
\hline BCM-4195 & $0 / 2(0)$ & 0 & $0 / 2(0)$ & 0 & 0 \\
\hline BCM-4272* & $3 / 3(100)$ & $<1-25$ & $4 / 6(67)^{c l}$ & $<1-132$ & 29 \\
\hline BCM-4664* & 1/3 (33) & 2 & ND & ND & 0 \\
\hline BCM-4888* & $5 / 5(100)^{\mathrm{Cl}}$ & $3-28$ & $0 / 4(0)$ & 0 & 67 \\
\hline BCM-5097 & $3 / 6(50)^{C l}$ & $10-91$ & $3 / 5(60)$ & $12-30$ & 36 \\
\hline BCM-5156 & $3 / 5(60)$ & $1-28$ & $1 / 2(50)$ & 2 & 0 \\
\hline BCM-5438 & $2 / 2(100)$ & $2-3$ & $2 / 2(100)$ & $4-67$ & 0 \\
\hline BCM-5471 & $3 / 6(50)^{c 1}$ & $1-10$ & $2 / 4(50)^{C l}$ & $<1-6$ & 33 \\
\hline BCM-5998 & $5 / 7(71)$ & $<1-27$ & $6 / 8(75)^{C l}$ & $1-40$ & 0 \\
\hline BCM-6257 & $3 / 3(100)^{C l}$ & $3-4$ & $2 / 3(67)^{\mathrm{Cl}}$ & $22-39$ & 6 \\
\hline
\end{tabular}


The data are presented in a collapsed $2 \times 2$ contingency table without different strata representing different PDX lines. The association between the ability to produce CTCs or CTC cluster occurrence and the presence of LM was assessed within each line because the latter was evaluated in a previous study [26]. These associations were assessed by Fisher's Exact test, and data were represented in $2 \times 2$ contingency table. To derive genetic signatures associated with CTC clusters and LM, genes that were differentially expressed and had some statistical evidence of being repeatable were considered; this signature was determined by using a linear contrast $t$ test $\mathrm{q}$-value (also called false discovery rate (FDR) $<0.25$ or at least 16 -fold average difference in expression between LM-positive and LM-negative PDX lines, which corresponds to a $\log 2$ contrast between LM-positive and LM-negative scoring greater than 4 in absolute value. To determine the prognostic value of the four-gene profile, geneexpression data of human $\mathrm{BC}$ [28] were each scored, taking the sum of the two "upregulated" genes minus the sum of the two "downregulated" genes (by using z-normalized expression values). All $P$ values reported were two-sided, unless otherwise specified.

\section{Results}

\section{BC PDX line characteristics}

We used 18 PDX-bearing mouse models, which have been described in detail previously [26]. Sixteen of these lines $(89 \%)$ were established by using primary breast tumor fragments obtained from patients without metastases. The other two lines (BCM-3561 and BCM-3613) were developed by transplanting tumor cells isolated from ascites and pleural fluid collected from two patients with metastatic disease, respectively. Among the lines we screened, one line (BCM-5097) was estrogen receptor (ER)-positive, progesterone receptor (PR)positive, but human epidermal growth factor receptor 2 (HER2) negative; three lines were ER-negative and PRnegative, but HER2-positive (BCM-3143, BCM-3613, BCM-3963); one line was positive for ER, PR, and HER2 (BCM-4888); the remaining 13 lines (72\%) were triple (ER, PR, HER2)-negative. All the tumor transplants were positive for either CK19 or CK5/6 or both, as previously reported [26].

\section{Detection of CTCs, BM-DTCs, and LM in BC PDX lines}

All five age-matched control female mice were negative for both CTCs and BM-DTCs. Individual CTC and BMDTC detection data in all tested mice are reported in Additional file 1: Table S1. Of 81 mice, only eight did not have adequate sample for CTC analysis and are labeled as NA (not available). The DTC analysis was initiated later when we already had collected CTC data for $>20$ mice. Therefore, DTC data were not available for a total of 28 mice because of either inadequate or no bone marrow sample.

Among the 18 PDX lines screened, we detected CTCs in $15(83 \%)$ (Table 1). The rate of CTC detection, defined as number of mice positive for CTCs divided by total number of mice tested, for CTC-positive PDX lines ranged from $17 \%$ to $100 \%$. CTCs were identified either as individual cells or as clusters of cells that were pancytokeratin positive (Figure 1). We found CTC clusters in six of the 18 PDX lines (33\%) (Table 1); of these, four were triple-negative (BCM-3204, BCM-3887, BCM-5471, BCM-6257), one was ER/PR/HER2-positive (BCM-4888), and one was ER/PR-positive and HER2-negative (BCM5097). The maximum number of CTCs detected in a single mouse was 92 per $20 \mu \mathrm{l}$ of blood, whereas a maximum of 20 to 25 cells was found within a CTC cluster.

BM-DTCs were found in 10 of the 16 PDX lines examined (62.5\%). The rate of BM-DTC detection ranged from $20 \%$ to $100 \%$ in the BM-DTC-positive lines (Table 1). Similar to CTCs, BM-DTCs were identified as individual cells or as clusters (Figure 1). We found that four of 16 (25\%) lines had the presence of BM-DTC clusters (Table 1). Two lines (BCM-5471 and BCM-6257) had both CTC and BMDTC clusters. The highest number of BM-DTCs detected in the bone marrow of a single mouse was 132 per 2 million cells.

LMs, as previously reported [26], were detected in $50 \%$ (nine of 18) of PDX lines (Table 1); the detection rate in the LM-positive lines ranged from $6 \%$ to $67 \%$.

\section{Correlation between the presence of CTCs and BM-DTCs and LM}

In total, 81 mice were screened in this study (see Additional file 1: Table S1). Of these, 46 mice had both CTCs and BMDTCs evaluated. The presence of CTCs was strongly associated with the presence of BM-DTCs $(P=0.0047$, Fisher's Exact test, Table 2). Even when the data were adjusted for PDX line by using the Cochran-Mantel-Haenszel test, the association was still significant $(P=0.0364)$.

The LM detection rates in these PDX lines were available from the previously conducted studies and hence were evaluated per PDX line rather than per individual mouse [26]. Remarkably, of the nine PDX lines positive for LM, all had detectable CTCs. Conversely, CTCs were detected in only six of the nine lines without LM. Overall, among the 13 PDX lines that had detectable CTCs and had both BM-DTCs and LM rates available, all the lines had either BM-DTCs or LM or both (Table 1). In turn, of the 14 lines with BM-DTCs and/or LM, only one did not have detectable CTCs. Despite this high concordance, the presence of CTCs in the 18 PDX lines we screened did not significantly correlate with the occurrence of LM previously reported in the same lines [26] ( $P=0.46$, Fisher's Exact test). Interestingly however, 


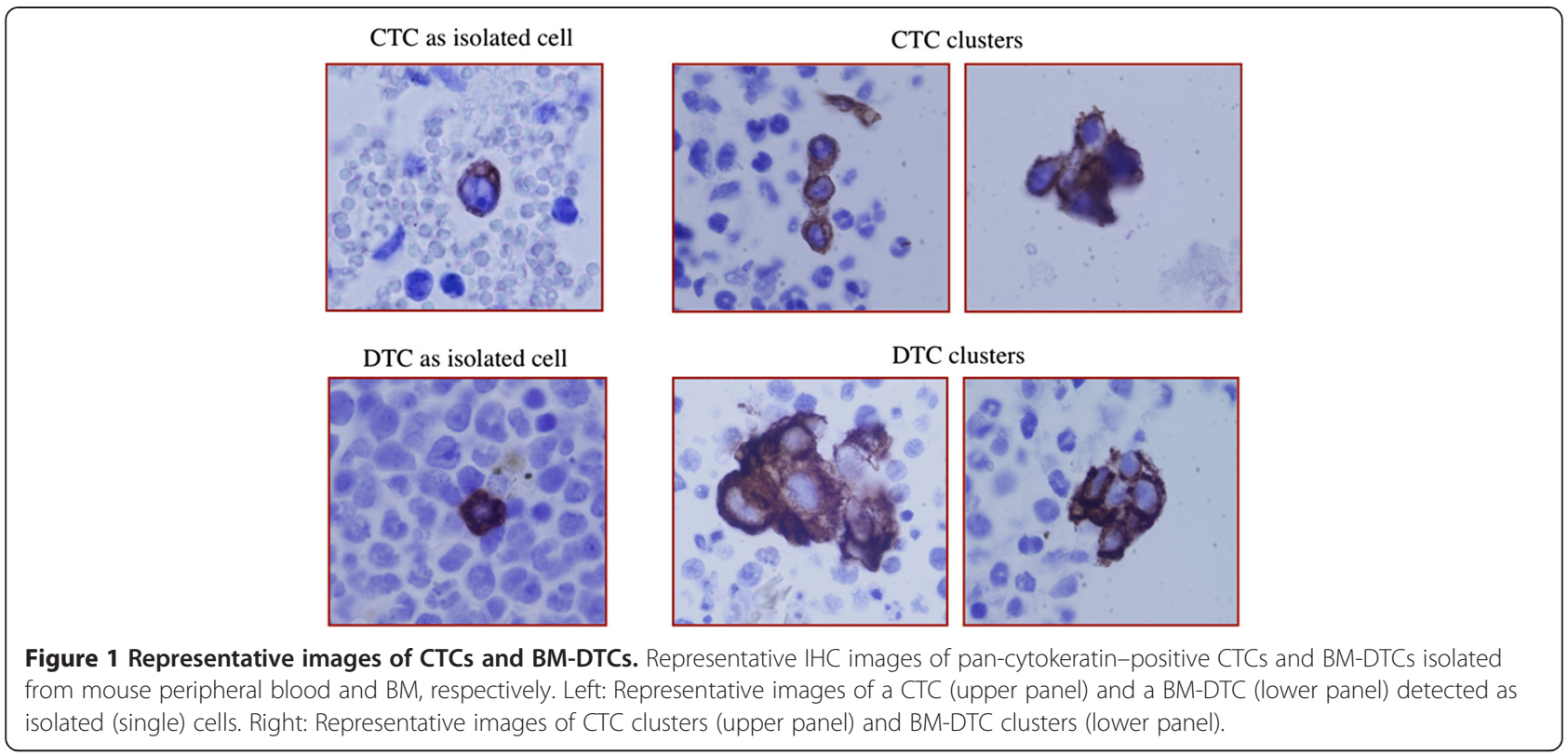

the detection of CTC clusters was highly associated with lung metastatic potential $(P=0.009$, Fisher's Exact test, Table 3). All six lines in which CTC clusters were found also had developed LM (Table 1). When the analysis was restricted to the 13 lines with triple negative $\mathrm{BC} P D X$, this association was also significant $(P=0.007$, Fisher's Exact test).

\section{Genetic signature of primary tumor associated with CTCs clusters and LM}

Because of the association between the presence of CTC clusters and LM, we wanted to understand the genetic signatures of the primary PDX tumors that give rise to CTC clusters and LM. We computed two separate genetic signatures associated with the presence of CTC clusters and with lung metastatic potential by using the gene-expression arrays of primary tumors from different PDX lines. We identified a set of 35 genes, which formed the CTC cluster-associated signature (see Additional file 2: Table S2). The LM-associated gene signature included 34 genes (Additional file 2: Table S3). Overlapping these two

Table 2 Correlation between the presence of CTCs and BM-DTCs in BC PDX-bearing mice

\begin{tabular}{lll}
\hline & $\begin{array}{l}\text { Number of mice } \\
\text { with BM-DTCs }\end{array}$ & $\begin{array}{l}\text { Number of mice } \\
\text { without BM-DTCs }\end{array}$ \\
\hline Number of mice with CTCS & 16 & 13 \\
Number of mice without CTCs & 2 & 15 \\
\hline
\end{tabular}

The data are presented in the $2 \times 2$ collapsed table, ignoring the strata representing different PDX lines. $P=0.0047$; Fisher's Exact test and $P=0.0364$; Cochran-Mantel-Haenszel test, adjusting for PDX line.

BC, breast cancer; BM-DTC, disseminated tumor cells in bone marrow; CTC, circulating tumor cell; PDX, patient-derived xenograft. gene signatures resulted in a four-gene profile (Table 4), which was associated with a modest but significant reduction in distant metastases-free survival in a large compendium of publicly available datasets of early BC patients (log-rank $P=0.048,10$-year survival probability of $67 \%$ for patients in the top $33 \%$ of signature scores versus $73 \%$ for the rest of the patients) (Figure 2). This gene profile included two genes (HLA-DP1A and GJA1) that were downregulated and two genes (PEG3 and XIST) that were upregulated (Table 4).

\section{Discussion}

Multiple lines of evidence suggest that CTCs and BMDTCs can be used to study the metastatic process and be evaluated in "real-time" to monitor the molecular changes in progressing tumors. However, their use has been largely limited because of challenges in their isolation as well as the very low yield of cells detected in human subjects, especially in the early stages of disease. As an alternative strategy, we established the conditions for the detection of human CTCs and BM-DTCs in unique BC PDX models in this study. As previously shown, these preclinical models accurately resemble their

Table 3 Correlation between the presence of CTC clusters and $L M$ in BC PDX-bearing mice

\begin{tabular}{lll}
\hline & $\begin{array}{l}\text { Number of lines } \\
\text { with LM }\end{array}$ & $\begin{array}{l}\text { Number of lines } \\
\text { without LM }\end{array}$ \\
\hline Number of lines with CTC clusters & 6 & 0 \\
Number of lines without CTC clusters & 3 & 9 \\
\hline
\end{tabular}

$P=0.009$; Fisher's Exact test.

$\mathrm{BC}$, breast cancer; CTC, circulating tumor cell; LM, lung metastases; PDX, patient-derived xenograft. 
Table 4 Gene profile of BC PDX primary tumors associated with CTC clusters and LM

\begin{tabular}{lll}
\hline Genes & $\begin{array}{l}\text { Average fold change in } \\
\text { lines with CTC clusters } \\
\text { versus no CTC clusters }\end{array}$ & $\begin{array}{l}\text { Average fold change } \\
\text { in lines with LM versus } \\
\text { no LM }\end{array}$ \\
\hline HLA-DP1A* & 0.05 & 0.02 \\
GJA1* & 0.04 & 0.03 \\
PEG3^ & 42.42 & 25.63 \\
XIST^ & 17.03 & 43.5 \\
\hline
\end{tabular}

*Downregulated genes; $\wedge$ Upregulated genes.

BC, breast cancer; CTC, circulating tumor cell; LM, lung metastasis; PDX patient-derived xenograft.

parental human tumors in their molecular features and biologic behavior [26]. Important in this study, we have found that BC PDX models can provide a continuous and renewable source of human CTCs and BM-DTCs. In support of our findings in $\mathrm{BC}$, a recently published study showed that CTCs isolated from pancreatic adenocarcinoma PDX-bearing mice also represent a reliable tool to predict and monitor treatment response [29].

The rate of CTC and DTC detection $(83 \%$ and $62.5 \%$, respectively) in our studies with PDX models is higher than that reported in the literature for non-metastatic breast cancer patients. We attribute this to the differences in the CTC/DTC detection methods as well as evaluation of larger blood and bone marrow volume relative to the body size of PDX mice than that of patients, as elaborated here. First, most of the commercial

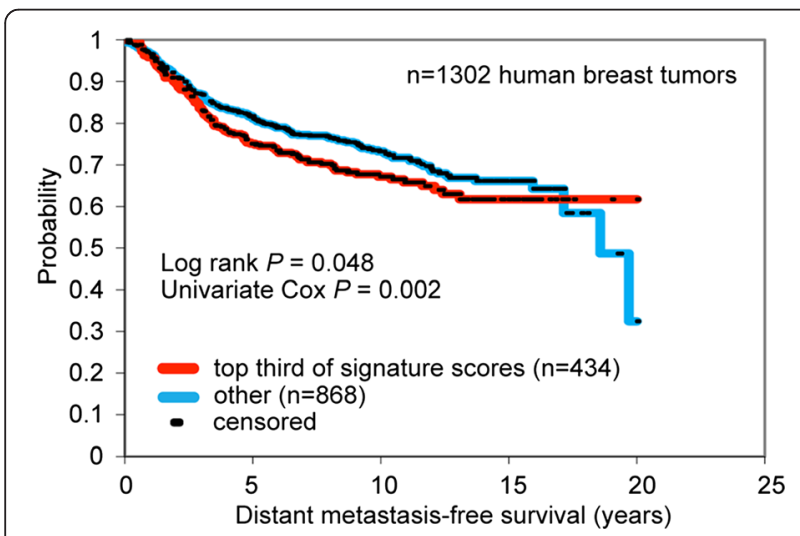

Figure 2 Prognostic value of the four-gene profile of BC PDXs associated with CTC clusters and LM. Four genes were found to overlap between two genetic signatures of primary tumor associated with CTC clusters and with lung metastases (LMs). Gene-expression profiles of human BCs were each scored for this signature (the score representing the values of the "high" genes minus the values of the "low" genes). Kaplan-Meier curves compare distant metastasis-free survival in $\mathrm{BC}$ patients with relatively higher signature scoring versus those with lower scoring. Univariate Cox evaluates the gene-signature score as a continuous variable. Patient data were extracted from publicly available datasets [28]. techniques to detect CTCs have relied on the presence of a limited number of epithelial markers (that is, epithelial cell adhesion molecule (EpCAM) and/or "epithelial" cytokeratins $8,18,19)$ [30]. This approach likely omits CTCs with a predominant mesenchymal phenotype and a lack of the epithelial markers [25,31-33]. In our approach for CTC/DTC detection, we used a quantitative immunohistochemistry assay to examine "human" tumor cells for the expression of multiple cytokeratin subtypes. Indeed, the pan-cytokeratin antibodies (AE1/AE3) we used bind to multiple cytokeratins present on both human epithelial and mesenchymal cells $[34,35]$. Second, the higher CTC/ DTC detection rate may also result from accessibility to large amounts of peripheral blood and bone marrow, relative to the small size of the mouse body. This is in contrast with only small blood and bone marrow volume used to assess CTC and DTCs in patients. These factors, as well as the immunodeficiency status of the PDX models, may contribute to the higher CTC/DTC detection rates we find in PDX models. In future, it will be of interest to compare CTCs/DTCs from patients side-by-side with those derived from matched PDXs as well as to compare characteristics of CTCs/DTCs within various immunodeficiency models and versus those with reconstituted immune components [36].

The observation of clusters of CTCs and BM-DTCs in the $\mathrm{BC}$ PDX-bearing mice is of great interest and further justifies the use of our PDX lines as clinically representative models to study these cells. Several studies have identified multicellular CTC clusters in BC $[37,38]$ and other types of cancer patients [39-43]. In a recent study, CTC clusters were found in $26 \%$ of patients with small cell lung cancer, and their presence was an independent prognostic factor [39]. Moreover, CTC clusters isolated from $\mathrm{BC}$ patients had high expression of mesenchymal markers and relatively low expression of epithelial markers, suggesting a potential link between the generation of CTC clusters and the EMT process [33]. However, the role of CTC clusters in cancer metastatic dissemination remains unclear. In our study, we found a significant association between the presence of CTC clusters and lung metastatic potential. Only one other study, to our knowledge, has shown a similar association in patients with clear cell renal cell carcinoma [41]. The infrequent finding of clusters of CTCs and BM-DTCs in other studies may be related to the isolation and detection techniques. It is possible that our method of processing the blood and bone marrow fractions may facilitate the detection of clusters.

Of note, we found variability in the detection of CTCs and BM-DTCs in different mice within the same PDX line. The same variability was also present in the previously reported LM detection among these lines [26]. This variability may be attributed to the intratumoral 
heterogeneity that is commonly seen in patients or some host-specific factors that may influence tumor initiation and progression. However, this observation emphasizes that the future studies to understand the influence of CTCs in distant metastases should include the analysis at a mouse level as well as an overall analysis for the PDX line. In addition, our finding of a significant correlation between the presence of CTCs and BM-DTCs within the same mice is consistent with what is observed in early BC patients [17]. All the BC PDX lines that had CTCs also had BM-DTCs and/or LM. This high concordance rate suggests that these CTCs and BM-DTCs are early indicators of metastatic potential and as such are important for molecular characterization and tumor biology studies. Because BM-DTCs were not evaluated in sufficient numbers of mice for most lines, correlation analyses as well as gene-expression analyses in the PDX lines were restricted to only the CTCs and LM data in this study. Future studies to characterize molecular profiles of CTCs, BM-DTCs, and lung lesions may uncover important biomarkers and treatment targets to prevent metastases.

The four-gene profile we obtained by overlapping the two genetic signatures of primary PDX tumors associated with CTC clusters and with LM was associated with a significant reduction in distant metastases-free survival in early BC patients. However, the observed association was weak because of limitations such as sample size and confounding variables, such as different subtypes and various treatments. Future studies using additional PDX lines are also necessary to validate this gene profile. In support of the derived gene profile, however, other reports have independently identified some of these genes to be associated with LM in BC. For example, HLA$D P 1 A$, which encodes a transmembrane protein involved in the antigen-presentation process, was downregulated in both the CTC clusters and LM signatures. Interestingly, $H L A-D P 1 A$ was one of the genes downregulated in the LM signatures generated in two independent studies in BC [44]. The other downregulated gene in our four-gene set was GJA1, which encodes a major protein in gap junctions. GJA1, also known as connexin 43 (Cx43), has been shown to suppress mammary tumor metastasis to the lung in a mouse model [45]. Specifically, missense mutation (G60S) in this gene led to production of an altered $\mathrm{Cx} 43$ protein that acts in a dominant-negative fashion to disrupt gap-junction assembly and function. This mutation was associated with higher rates of LM in ErbB2-overexpressing mice. Of the two upregulated genes in our four-gene profile, XIST is a noncoding RNA gene on X chromosome and plays a major role in $\mathrm{X}$ inactivation. Although overexpression of XIST has been seen in BRCA-1-associated BCs, which typically metastasizes to lungs $[46,47]$, a direct link between XIST and LM in BC has not been established. The other upregulated gene, $P E G 3$, encodes a $\mathrm{C} 2 \mathrm{H} 2$ type zinc finger protein implicated in regulation of body temperature, feeding behavior, and obesity [48], as well as growth, apoptosis, and maternal nurturing behavior [49]. The role of PEG3 in BC and LM is not clear and warrants further investigation.

\section{Conclusion}

The analysis of CTCs and BM-DTCs in the clinical setting is challenging and imposes multiple limitations. In this study, we provide the first evidence that BC PDX models represent a novel and promising experimental resource for investigating the role of CTCs and BMDTCs in promoting overt metastases in $\mathrm{BC}$ and for their characterization to identify new treatment targets.

\section{Additional files}

Additional file 1: Table S1. Individual mouse data for CTCS and BM-DTCs for all PDX lines screened.

Additional file 2: Tables S2 and S3. S2. Gene signature of BC PDX primary tumors associated with the presence of CTC clusters. S3. Gene signature of BC PDX primary tumors associated with the presence of lung metastases.

\section{Abbreviations}

BC: Breast cancer; BM-DTC: bone marrow disseminated tumor cells; CTC: circulating tumor cells; Cx43: Connexin 43; EDTA: ethylenediaminetetraacetic acid; EMT: epithelial-to-mesenchymal transition; EpCAM: epithelial cell adhesion molecule; ER: estrogen receptor; FDR: false discovery rate; HER2: human epidermal growth factor receptor 2; IHC: immunohistochemistry; LIMMA: LInear Models for MicroArray data; LM: lung metastases; PBS: phosphate-buffered saline;

PDX: patient-derived xenograft; PR: progesterone receptor; RBC: red blood cell.

\section{Competing interests}

Mike T. Lewis is a member of StemMed Holdings LLC, and a limited partner in StemMed Ltd. The following authors declare no competing interests: Mario Giuliano, Sabrina Herrera, Pavel Christiny, Chad Shaw, Chad J. Creighton, Tamika Mitchell, Raksha Bhat, Xiaomei Zhang, Sufeng Mao, Lacey E. Dobrolecki, Ahmed Al-rawi, Fengju Chen, Bianca M. Veneziani, Xiang H. Zhang, Rinath M. Jeselsohn, Susan G. Hilsenbeck, Alejandro Contreras, Carolina Gutierrez, Mothaffar F. Rimawi, C. Kent Osborne, Rachel Schiff, and Meghana V. Trivedi.

\section{Authors' contributions}

MG collected and processed blood and bone marrow samples, conceived of and designed the study, and drafted the manuscript. SH performed pathology analysis. PC participated in collection and processing of blood and bone marrow samples. CS performed bioinformatics analysis, participated in data interpretation, and helped to draft the manuscript. CJC participated in bioinformatics analysis and data interpretation, and helped to draft the manuscript. TM participated in accomplishment of in vivo studies and collection of blood and bone marrow samples. RB participated in processing of blood and bone marrow samples. XZ coordinated the in vivo studies. SM processed blood and bone marrow samples. LED participated in accomplishment of in vivo studies. AA participated in pathology data analysis. FC participated in bioinformatics analysis and data interpretation. BMV participated in interpretation of data and study design. XHZ participated in interpretation of data and helped to draft the manuscript. RMJ participated in study design and interpretation of data. SGH performed statistical analysis and participated in interpretation of data. AC participated in pathology analysis and data interpretation. CG coordinated pathology analyses. M.F.R. participated in study design and data interpretation. CKO participated in study coordination and helped to draft the manuscript. MTL participated in study design, provided 
in vivo PDX mouse models, and helped to draft the manuscript. RS conceived the study, participated in its design and coordination, and helped to draft the manuscript. MVT conceived of, designed, and coordinated the study, performed data analysis and interpretation, and drafted the manuscript. All authors read and approved the final manuscript.

\section{Acknowledgements}

This study was supported in part by National Institutes of Health grants U54CA149196 (to R.S. and M.T.); P01 SPORE (to R.S. and R.M.J.); faculty start-up funds (to M.T.); Cancer Prevention and Research Institute of Texas (CPRIT) program RP101499, Baylor College of Medicine Comprehensive Cancer Training Program (to M.G.); Susan G. Komen grant KG120001 (to M.L.) Dan L. Duncan Cancer Center Grant P30CA125123 from the National Cancer Institute, Cytometry and Cell Sorting Core at Baylor College of Medicine with funding from the NIH (P30 Al036211, P30 CA125123, and S10 RR024574), research grants from the Breast Cancer Research Foundation; the Entertainment Industry Foundation/Lee Jeans, and SU2C Breast Cancer Program.

We also acknowledge the following Baylor College of Medicine shared resources: Biostatistics \& Informatics; Cytometry and Cell Sorting; and Human Tissue Acquisition and Pathology.

\section{Author details}

'Lester and Sue Smith Breast Center, Baylor College of Medicine, Houston, TX, USA. '2Department of Clinical Medicine and Surgery, University Federico II, Naples, Italy. ${ }^{3}$ Department of Pharmacy Practice and Translational Research, University of Houston, Houston, TX, USA. ${ }^{4}$ Dan L. Duncan Cancer Center, Baylor College of Medicine, Houston, TX, USA. ${ }^{5}$ Department of Molecular and Human Genetics, Baylor College of Medicine, Houston, TX, USA. ${ }^{6}$ Department of Medicine, Baylor College of Medicine, Houston, TX, USA. " Department of Molecular Medicine and Medical Biotechnology, University Federico II, Naples, Italy. ${ }^{8}$ Department of Molecular and Cellular Biology, Baylor College of Medicine, Houston, TX, USA. ${ }^{9}$ Department of Pathology, Baylor College of Medicine, Houston, TX, USA. ${ }^{10}$ Dana-Farber Cancer Institute, Harvard Medical School, Boston, MA, USA. ${ }^{11}$ Department of Radiology, Baylor College of Medicine, Houston, TX, USA. ${ }^{12}$ Department of Pharmacological and Pharmaceutical Sciences, University of Houston, Houston, TX, USA.

Received: 10 June 2014 Accepted: 18 December 2014 Published online: 09 January 2015

\section{References}

1. Allard WJ, Matera J, Miller MC, Repollet M, Connelly MC, Rao C, et al. Tumor cells circulate in the peripheral blood of all major carcinomas but not in healthy subjects or patients with nonmalignant diseases. Clin Cancer Res. 2004;10:6897-904.

2. Sun YF, Yang XR, Zhou J, Qiu SJ, Fan J, XU Y. Circulating tumor cells: advances in detection methods, biological issues, and clinical relevance. J Cancer Res Clin Oncol. 2011;137:1151-73.

3. Paget $\mathrm{S}$. The distribution of secondary growths in cancer of the breast. Lancet. 1889;133:571-3.

4. Cristofanilli M, Budd GT, Ellis MJ, Stopeck A, Matera J, Miller MC, et al. Circulating tumor cells, disease progression, and survival in metastatic breast cancer. N Engl J Med. 2004;351:781-91.

5. Xenidis N, Ignatiadis M, Apostolaki S, Perraki M, Kalbakis K, Agelaki S, et al. Cytokeratin-19 mRNA-positive circulating tumor cells after adjuvant chemotherapy in patients with early breast cancer. J Clin Oncol. 2009;27:2177-84.

6. Bidard FC, Mathiot C, Delaloge S, Brain E, Giachetti S, de Cremoux P, et al. Single circulating tumor cell detection and overall survival in nonmetastatic breast cancer. Ann Oncol. 2010;21:729-33.

7. Cristofanilli M, Hayes DF, Budd GT, Ellis MJ, Stopeck A, Reuben JM, et al. Circulating tumor cells: a novel prognostic factor for newly diagnosed metastatic breast cancer. J Clin Oncol. 2005;23:1420-30.

8. Hayes DF, Cristofanilli M, Budd GT, Ellis MJ, Stopeck A, Miller MC, et al. Circulating tumor cells at each follow-up time point during therapy of metastatic breast cancer patients predict progression-free and overall survival. Clin Cancer Res. 2006:12:4218-24.

9. Budd GT, Cristofanilli M, Ellis MJ, Stopeck A, Borden E, Miller MC, et al. Circulating tumor cells versus imaging-predicting overall survival in metastatic breast cancer. Clin Cancer Res. 2006;12:6403-9.
10. Liu MC, Shields PG, Warren RD, Cohen P, Wilkinson M, Ottaviano $Y L$, et al. Circulating tumor cells: a useful predictor of treatment efficacy in metastatic breast cancer. J Clin Oncol. 2009;27:5153-9.

11. Alunni-Fabbroni M. Circulating tumor cells in clinical practice: methods of detection and possible characterization. Methods. 2010;50:289-97.

12. Bidard FC, Peeters DJ, Fehm T, Nole F, Gisbert-Criado R, Mavroudis D, et al. Clinical validity of circulating tumour cells in patients with metastatic breast cancer: a pooled analysis of individual patient data. Lancet Oncol. 2014;15:406-14.

13. Riethdorf S, Muller V, Zhang L, Rau T, Loibl S, Komor M, et al. Detection and HER2 expression of circulating tumor cells: prospective monitoring in breast cancer patients treated in the neoadjuvant GeparQuattro trial. Clin Cancer Res. 2010;16:2634-45.

14. Gradilone A, Naso G, Raimondi C, Cortesi E, Gandini O, Vincenzi B, et al. Circulating tumor cells (CTCs) in metastatic breast cancer (MBC): prognosis, drug resistance and phenotypic characterization. Ann Oncol. 2011;22:86-92.

15. Fehm T, Muller $V$, Aktas B, Janni W, Schneeweiss A, Stickeler E, et al. HER2 status of circulating tumor cells in patients with metastatic breast cancer: a prospective, multicenter trial. Breast Cancer Res Treat. 2010;124:403-12.

16. Hayes DF, Smerage J. Is there a role for circulating tumor cells in the management of breast cancer? Clin Cancer Res. 2008;14:3646-50.

17. Fehm T, Hoffmann O, Aktas B, Becker S, Solomayer EF, Wallwiener D, et al. Detection and characterization of circulating tumor cells in blood of primary breast cancer patients by RT-PCR and comparison to status of bone marrow disseminated cells. Breast Cancer Res. 2009:11:R59.

18. Pantel K, Alix-Panabieres C, Riethdorf S. Cancer micrometastases. Nat Rev Clin Oncol. 2009;6:339-51.

19. Riethdorf S, Wikman H, Pantel K. Review: biological relevance of disseminated tumor cells in cancer patients. Int J Cancer. 2008;123:1991-2006.

20. Hall C, Krishnamurthy S, Lodhi A, Bhattacharyya A, Anderson A, Kuerer H, et al. Disseminated tumor cells predict survival after neoadjuvant therapy in primary breast cancer. Cancer. 2012;118:342-8.

21. Janni W, Vogl FD, Wiedswang G, Synnestvedt M, Fehm T, Juckstock J, et al. Persistence of disseminated tumor cells in the bone marrow of breast cancer patients predicts increased risk for relapse: a European pooled analysis. Clin Cancer Res. 2011;17:2967-76.

22. Gorges TM, Pantel K. Circulating tumor cells as therapy-related biomarkers in cancer patients. Cancer Immunol Immunother. 2013;62:931-9.

23. Lianidou ES, Mavroudis D, Georgoulias V. Clinical challenges in the molecular characterization of circulating tumour cells in breast cancer. Br J Cancer. 2013:108:2426-32.

24. Alix-Panabieres C, Riethdorf S, Pantel K. Circulating tumor cells and bone marrow micrometastasis. Clin Cancer Res. 2008;14:5013-21.

25. Gorges TM, Tinhofer I, Drosch M, Rose L, Zollner TM, Krahn T, et al. Circulating tumour cells escape from EpCAM-based detection due to epithelial-to-mesenchymal transition. BMC Cancer. 2012;12:178.

26. Zhang X, Claerhout S, Prat A, Dobrolecki LE, Petrovic I, Lai Q, et al. A renewable tissue resource of phenotypically stable, biologically and ethnically diverse, patient-derived human breast cancer xenograft models. Cancer Res. 2013;73:4885-97.

27. Park J, Al-Ramahi I, Tan Q, Mollema N, Diaz-Garcia JR, Gallego-Flores T, et al. RAS-MAPK-MSK1 pathway modulates ataxin 1 protein levels and toxicity in SCA1. Nature. 2013:498:325-31.

28. Kessler JD, Kahle KT, Sun T, Meerbrey KL, Schlabach MR, Schmitt EM, et al. A SUMOylation-dependent transcriptional subprogram is required for Myc-driven tumorigenesis. Science. 2012;335:348-53.

29. Torphy RJ, Tignanelli CJ, Kamande JW, Moffitt RA, Herrera Loeza SG, Soper SA, et al. Circulating tumor cells as a biomarker of response to treatment in patient-derived xenograft mouse models of pancreatic adenocarcinoma. PLoS One. 2014;9:e89474.

30. Alix-Panabieres C, Pantel K. Technologies for detection of circulating tumor cells: facts and vision. Lab Chip. 2013;14:57-62.

31. Aktas B, Tewes M, Fehm T, Hauch S, Kimmig R, Kasimir-Bauer S. Stem cell and epithelial-mesenchymal transition markers are frequently overexpressed in circulating tumor cells of metastatic breast cancer patients. Breast Cancer Res. 2009;11:R46

32. Kasimir-Bauer S, Hoffmann O, Wallwiener D, Kimmig R, Fehm T. Expression of stem cell and epithelial-mesenchymal transition markers in primary breast cancer patients with circulating tumor cells. Breast Cancer Res. 2012;14:R15. 
33. Kallergi G, Papadaki MA, Politaki E, Mavroudis D, Georgoulias V, Agelaki S. Epithelial to mesenchymal transition markers expressed in circulating tumour cells of early and metastatic breast cancer patients. Breast Cancer Res. 2011;13:R59.

34. Cates JM, Dupont WD, Barnes JW, Edmunds HS, Fasig JH, Olson SJ, et al. Markers of epithelial-mesenchymal transition and epithelial differentiation in sarcomatoid carcinoma: utility in the differential diagnosis with sarcoma. Appl Immunohistochem Mol Morphol. 2008;16:251-62.

35. Yamaguchi R, Tanaka M, Kondo K, Yokoyama T, Maeda I, Tsuchiya S, et al. Immunohistochemical study of metaplastic carcinoma and central acellular carcinoma of the breast: central acellular carcinoma is related to metaplastic carcinoma. Med Mol Morphol. 2012;45:14-21.

36. Liu X, Li H, Liu J, Guan Y, Huang L, Tang H, et al. Immune reconstitution from peripheral blood mononuclear cells inhibits lung carcinoma growth in NOD/SCID mice. Oncol Lett. 2014:8:1638-44.

37. Yu M, Bardia A, Wittner BS, Stott SL, Smas ME, Ting DT, et al. Circulating breast tumor cells exhibit dynamic changes in epithelial and mesenchymal composition. Science. 2013;339:580-4.

38. Cho EH, Wendel M, Luttgen M, Yoshioka C, Marrinucci D, Lazar D, et al. Characterization of circulating tumor cell aggregates identified in patients with epithelial tumors. Phys Biol. 2012;9:016001.

39. Hou JM, Krebs MG, Lancashire L, Sloane R, Backen A, Swain RK, et al. Clinical significance and molecular characteristics of circulating tumor cells and circulating tumor microemboli in patients with small-cell lung cancer. J Clin Oncol. 2012;30:525-32.

40. Stott SL, Hsu CH, Tsukrov DI, Yu M, Miyamoto DT, Waltman BA, et al, Isolation of circulating tumor cells using a microvortex-generating herringbone-chip. Proc Natl Acad Sci U S A. 2010;107:18392-7.

41. Kats-Ugurlu G, Roodink I, de Weijert M, Tiemessen D, Maass C, Verrijp K, et al. Circulating tumour tissue fragments in patients with pulmonary metastasis of clear cell renal cell carcinoma. J Pathol. 2009;219:287-93.

42. Molnar B, Ladanyi A, Tanko L, Sreter L, Tulassay Z. Circulating tumor cell clusters in the peripheral blood of colorectal cancer patients. Clin Cancer Res. 2001;7:4080-5

43. Brandt B, Junker R, Griwatz C, Heidl S, Brinkmann O, Semjonow A, et al. Isolation of prostate-derived single cells and cell clusters from human peripheral blood. Cancer Res. 1996:56:4556-61.

44. Minn AJ, Gupta GP, Siegel PM, Bos PD, Shu W, Giri DD, et al. Genes that mediate breast cancer metastasis to lung. Nature. 2005;436:518-24.

45. Plante I, Stewart MK, Barr K, Allan AL, Laird DW. CX43 suppresses mammary tumor metastasis to the lung in a Cx43 mutant mouse model of human disease. Oncogene. 2011;30:1681-92.

46. Kriege M, Seynaeve C, Meijers-Heijboer H, Collee JM, Menke-Pluymers MB, Bartels CC, et al. Distant disease-free interval, site of first relapse and postrelapse survival in BRCA1- and BRCA2-associated compared to sporadic breast cancer patients. Breast Cancer Res Treat. 2008;111:303-11.

47. Sirchia SM, Tabano S, Monti L, Recalcati MP, Gariboldi M, Grati FR, et al. Misbehaviour of XIST RNA in breast cancer cells. PLoS One. 2009;4:e5559.

48. Curley JP, Pinnock SB, Dickson SL, Thresher R, Miyoshi N, Surani MA, et al. Increased body fat in mice with a targeted mutation of the paternally expressed imprinted gene Peg3. FASEB J. 2005;19:1302-4.

49. Murphy SK, Wylie AA, Jirtle RL. Imprinting of PEG3, the human homologue of a mouse gene involved in nurturing behavior. Genomics. 2001;71:110-7.

\section{Submit your next manuscript to BioMed Central and take full advantage of:}

- Convenient online submission

- Thorough peer review

- No space constraints or color figure charges

- Immediate publication on acceptance

- Inclusion in PubMed, CAS, Scopus and Google Scholar

- Research which is freely available for redistribution 